\title{
CRITICAL DIGITAL ISCHEMIA IN SYSTEMIC SCLEROSIS EXACERBATED BY MULTIPLE MYELOMA - CASE REPORT
}

\author{
Andre Silva Franco ${ }^{1, \star}$, Gabriel Berlingieri Polho ${ }^{1}$, Thiago Cavalcanti Matos ${ }^{1}$, Ana Paula Luppino Assad ${ }^{1}$, Renata Miossi ${ }^{1}$, Percival \\ Degrava Sampaio-Barros ${ }^{1}$
}

1.Hospital das Clínicas da Faculdade de Medicina da USP, Universidade de São Paulo, São Paulo (SP), Brazil.

*Corresponding author: andre.franco@fm.usp.br

\section{BACKGROUND}

Literature on systemic sclerosis (SSc) and overlap with hematological malignancy is scarce. This case report presents a patient with SSc and multiple myeloma (MM), who had severe digital ischemia culminating in amputations of several fingers, probably due to paraprotein hyperviscosity with worsening of SSc vasculopathy.

\section{CASE REPORT}

A 65 year-old white female patient was diagnosed with limited SSc in 2002, smoldering MM IgG/kappa in 2017 with 18\% plasma cells in bone marrow, and liver cirrhosis in 2018. The first presentation of MM consisted of anemia, weight loss and a monoclonal peak in serum protein electrophoresis (1.9 g/dL), without bone or kidney lesions, but Raynaud's phenomenon (RP) was aggravated even with optimized pharmacological treatment. The healthcare team chose to treat MM with cyclophosphamide and dexamethasone in 2017, and repeated the treatment in 2020, in the context of worsening anemia, gamma globulin peak and RP. Liver disease was secondary to autoimmune hepatitis, leading to portal hypertension with esophageal varices, being classified as CHILD-Pugh A. Due to this comorbidity, she was not considered for bone marrow transplantation. Although the course of her SSc was mild, with RP and occasional digital ulcers, facial and palmar telangiectasias, no visceral impairment and negative specific SSc autoantibodies, her nailfold capillaroscopy showed an SD pattern. In 2021, she was admitted to the emergency room with dry ischemia of all fingers and toes (Figure 1) despite optimized therapy with calcium channel blocker (nifedipine $40 \mathrm{mg} / \mathrm{day}$ ) and phosphodiesterase 5 inhibitor (sildenafil $150 \mathrm{mg} / \mathrm{day}$ ), associated to visual blurring. The diagnostic hypothesis was a hyperviscosity syndrome associated with MM reactivation. The patient underwent plasmapheresis and restarted chemotherapy VCD (cyclophosphamide, dexamethasone and bortezomib). Despite the initial laboratory improvement, 19 fingers required amputation (Figure 2).

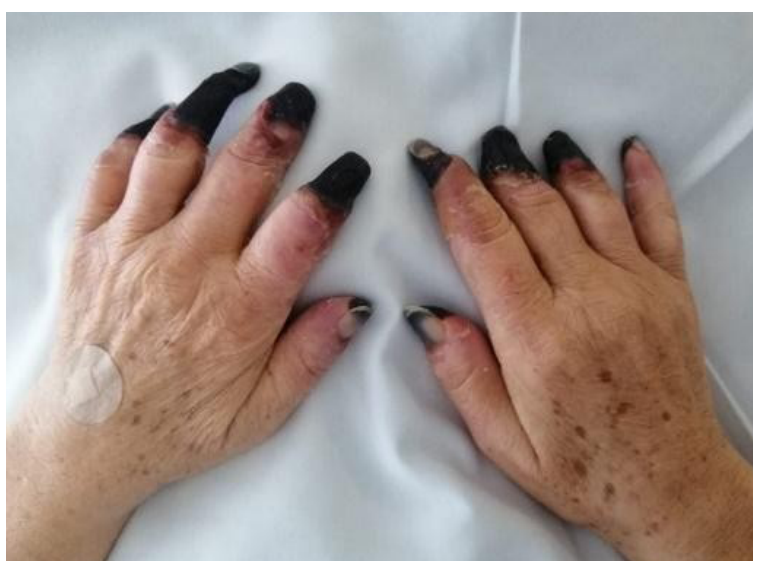

Figure 1. Digital ischemia.

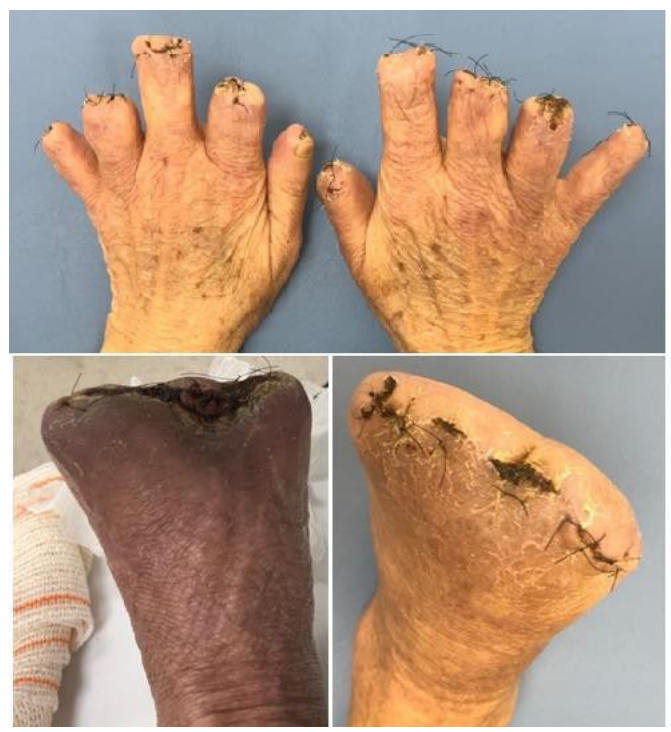

Figure 2. Hands and feet after amputation.

\section{CONCLUSION}

Although the association between SSc and MM is rare, it should be remembered in cases of significant worsening of RP, since a severe hyperviscosity syndrome can contribute to the amputation of extremities in these patients.

\section{KEYWORDS}

Systemic sclerosis, Multiple myeloma, Raynaud phenomenon. 